\title{
Over Pressure Risk Mitigation with SCADA in a Natural Gas Distribution System
}

\author{
Ghandi Rouainia $^{1, *}$, Mounira Rouainia $^{2}$, Abderrezak Metatla ${ }^{1}$ \\ ${ }^{1}$ Mechanical engineering and materials research laboratory, University of Skikda, Algeria \\ ${ }^{2} \mathrm{Chemical}$ engineering and environment research laboratory, University of Skikda, Algeria
}

Received November 6, 2019; Revised December 24, 2019; Accepted December 30, 2019

Copyright $\mathrm{O} 2020$ by authors, all rights reserved. Authors agree that this article remains permanently open access under the terms of the Creative Commons Attribution License 4.0 International License

\begin{abstract}
Natural gas distribution providers have periodic and reactive maintenance plans that are not providing enough feedback and data to establish proper predictive analysis for failure, especially on distribution gas regulator station that are regulating gas from transmission pressure to a safe customer distribution pressure, these stations are the only overpressure safeguard and the highest risk at the same time. With the recent advances in computer and telecommunications technologies, some major gas utilities initiated more robust predictive risk analysis plans by introducing SCADA supervisory control and data acquisition to gas distribution, mainly monitoring gas regulator stations and their behavior based on their established overpressure prevention philosophies and long-term predictive analysis goals. This study outlines a full SCADA monitoring solution on a dual run gas distribution regulator station equipped with primary overpressure protection and redundancy options. RTU (Remote Terminal Units) containing flow and pressure monitoring transmitters are installed at specific pressure reduction points in the station piping. Unlike common monitoring practice, we are introducing mid-stream pressure monitoring between the monitor and regular valves, filter differential and flow measurement in our proposed SCADA set up. This system provides real time visibility and data to the gas control room to quickly recognize abnormal or emergency events and assess the current health of regulator equipment. The outlined risk mitigations in this study are created based on identified risks of overpressure under several regulation failure modes of the regulators in standby and working modes. Our SCADA solution introduces means to enhance deductive analysis and predictability for gas control departments and monitoring groups leading to a safer and more reliable gas distribution system, improving emergency response and creating more comprehensive maintenance plans driven by long term predictive risk plans.
\end{abstract}

Keywords Natural Gas Processing, Overpressure Protection, Natural Gas Distribution Systems, SCADA,
Data Acquisition Systems, Natural Gas Regulator Stations, Risk Mitigation

\section{Introduction}

Distribution natural gas systems all over the world are served by multiple gas regulator stations forming hydraulically independent systems with redundancies and possible interconnections with manual and automated overpressure protection within the stations. The recent developments and the affordability in Supervisory Control and Data Acquisition (SCADA) implementations in utilities led to gas utilities adoption of these monitoring and control methods. These companies are facing challenging issues with aging facilities and increased demands driven by economic growth and global warming. Natural gas is still the most affordable way of heating homes in most developing countries.

The main risk facing gas utilities in their distribution systems is overpressure due to gas regulation failure. knowing that the only protection between the very high transmission pressure system is a distribution gas regulator station, all utilities created periodic and reactive maintenance plans that are established based on subject matter experts and facilities components manufacturers recommendations. These plans do not provide a mean to properly create real time system visibility and long-term predictive risk plans as well as establishing robust hydraulic simulation models based on real collected data.

Our SCADA solution focuses on real time visibility of regulator station functions and behavior to recognize abnormal and emergency events when they occur. This work was established based on similar studies in the city of Tokyo and SCADA systems in the gas industry research [1] [2].

Our main goal is to significantly reduce overpressure events thus reducing outages and recognizing abnormal 
and emergency events to improve field response and targeted and adequate response on pin-pointed issues locations.

Our SCADA solution allows constant assessment of the health of regulator equipment and creation of robust overpressure risk mitigation strategies based on collected pressure data and set alarms.

\section{The Gas Distribution SCADA Network}

The SCADA network [3] [4] is set up to provide a working operational interface to allow Gas Control operators to monitor in real time pressure, gas flow and water level alarms in distribution regulator stations installed underground in vaults.

This system creates on-demand supervisory controls when transmitted to the RTUs installed at each gas regulator station, shutoff gas flow and adjust operational conditions in hydraulic independent systems to suit the requirements of the moment. This relatively simple task involves the use of a wide range of technology and interfaces spanning large distances.

Looking at the functional block diagram in figure 1 , it is designed to show the physical connections and communications from the field to the gas control room. Where an RTU (Remote Terminal Unit) which is a computer capable of analyzing analog and digital inputs from pressure transmitters, filter differential transmitters and level switches will communicate data collected at key pressure reduction points on the station and send alarms to the Gas control room.

Key elements on the network in Figure 1 are:

- Engineering Workstations

- Operator Consoles

- Secure Database Servers

- Applications Station, Office Computers

- Secure Local Area Network

- Communications to field devices, RTUs (Remote Terminal Units)

- $\quad$ Radio Mountain Tops

We highly recommend Radio communication as more reliable to wireless cell and wired phone communications.

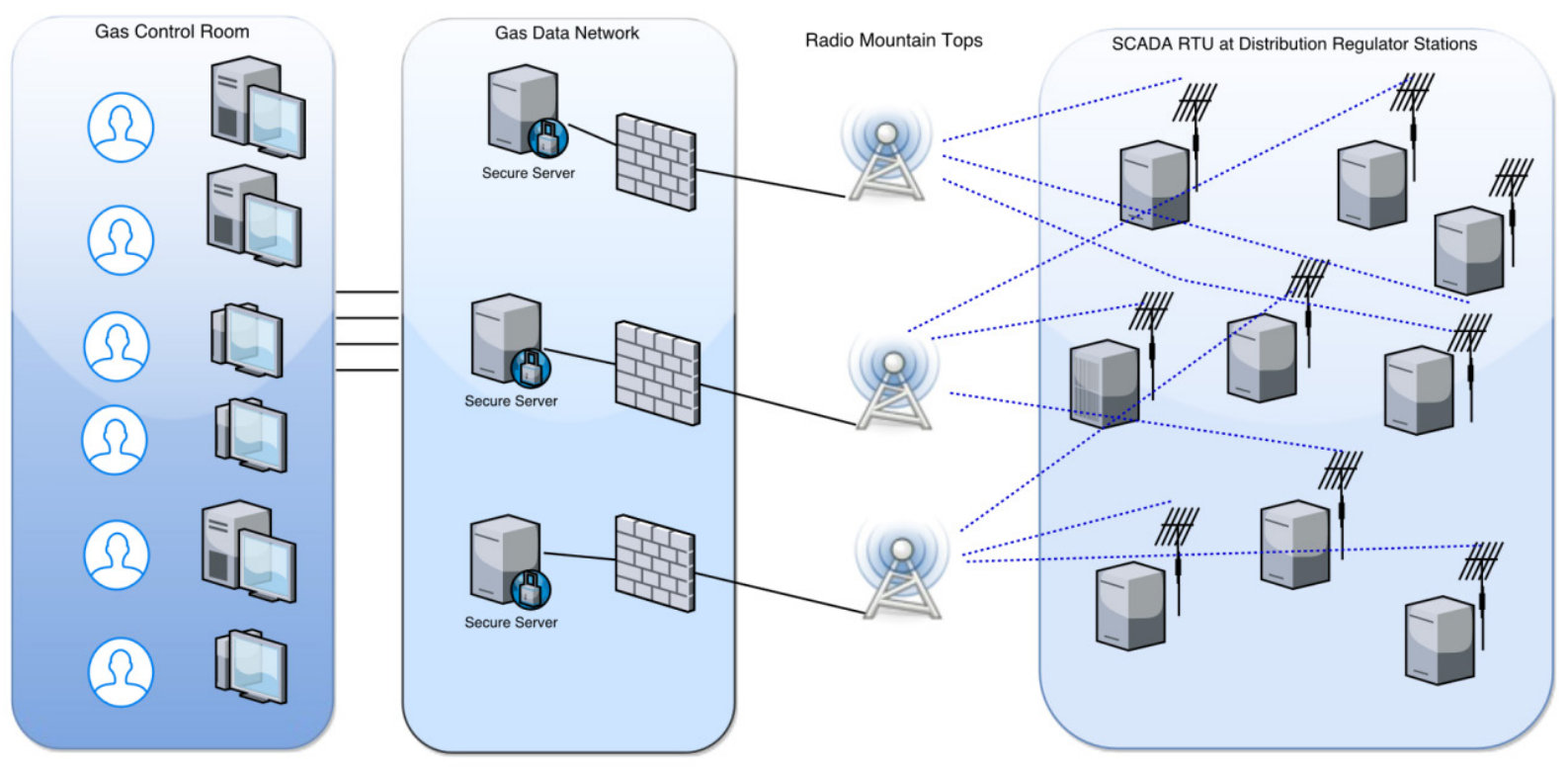

Figure 1. Gas Distribution SCADA Network Diagram 


\section{SCADA Monitoring Points on a Distribution Regulator Station}

Under normal conditions, the distribution regulator stations provide an operational function by controlling the line pressure downstream to ensure gas demand is satisfied. Under abnormal conditions, the stations provide a safety and protection function by ensuring that pressures are maintained within specified limits through the settings of the regulator set points.

From a monitoring function, since the regulator stations are the major control elements in the gas distribution system, the measurement of pressure upstream and downstream of the regulation station and flow through the station provides visibility on the state of the system as well as the behavior of the station equipment.

Our proposed high-pressure distribution regulator station has a typical outlet maximum allowable operating pressure of 60 PSIG and composed upstream to downstream of an inlet filter, an orifice type flow meter, a pressure standby or worker monitor, and a pressure regulator.

Intermediate pressure, downstream pressure, flow rate measurement, filter differential pressure, and dual liquid level switches are parameters evaluated as risk considerations for remote monitoring on the stations. Figure 2 depicts our proposed typical gas regulator station schematic and monitoring points, upstream, midstream on both primary, secondary (redundant) run and downstream monitoring.

Gas flow is measured on both runs downstream of the filter and upstream of the monitor. For our study we are using pilot operated Mooney Flowgrid regulating valves, they are the most commonly used regulators in North America in high pressure distribution stations.

Not shown in Figure 2, but may be added for the automation of the installations, is valve actuation for remote station shutoff when needed and water level switches installed at two different height levels, at station piping level and at regulators pilots level providing two levels of alarms.

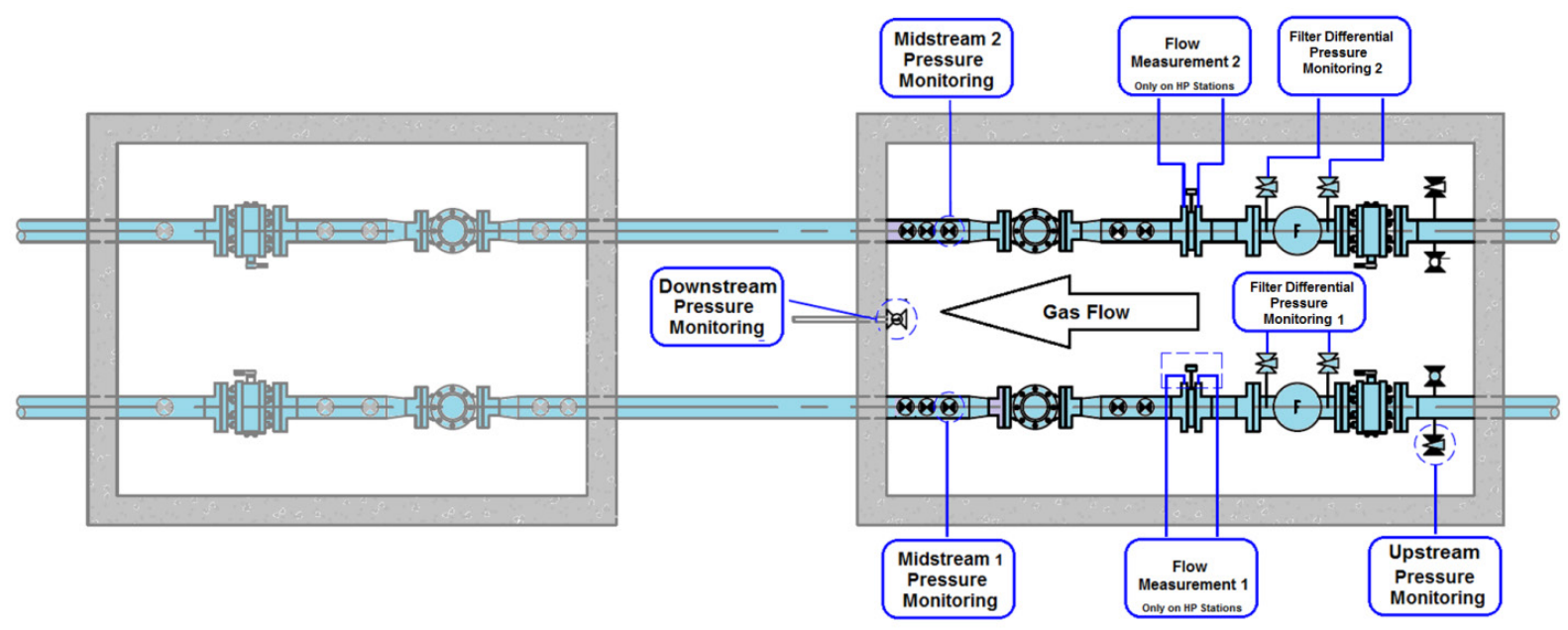

Figure 2. Typical below ground Regulator Station with SCADA Schematic. Primary and secondary runs are fed by the same inlet pipe 


\section{Risk Identification and SCADA Monitoring}

\subsection{Pressure Regulators with Standby Pressure Monitors}

Figure 3 depicts pilot operated Mooney Flowgrid regulators [5] installed in a regulator station with a standby pressure monitor and shows the active control of the pressure monitor when failure of the worker regulator occurs.

Our SCADA plan for monitoring pressure on regulator stations with standby monitors is to measure the upstream pressure, the intermediate pressure, and the downstream pressure. Measuring upstream and downstream pressures is a standard industry practice but our method includes the addition of intermediate pressure monitoring between monitor and regulator providing additional information to analyze the conditions of both regulating valves.

As shown in Figure 3 Under normal operational conditions, gas flows through both regulators in series and gas pressure is reduced across the worker regulator (line pressure of $110 \mathrm{psig}$ is reduced to $39 \mathrm{psig}$ ). In case of worker regulator failure, allowing downstream pressure to rise to the set pressure of the monitor, the monitor valve will begin to regulate pressure at or near the monitor pressure set point value (45 psig). The intermediate pressure between the two regulators will drop significantly (110 psig to $45 \mathrm{psig}$ ) to the monitor set point pressure value. In this scenario, Gas Control will recognize the alarm coming from the RTU monitoring the station showing that the pressure monitor is now in control and the regulator valve at this station requires immediate service and local technicians will be dispatched.

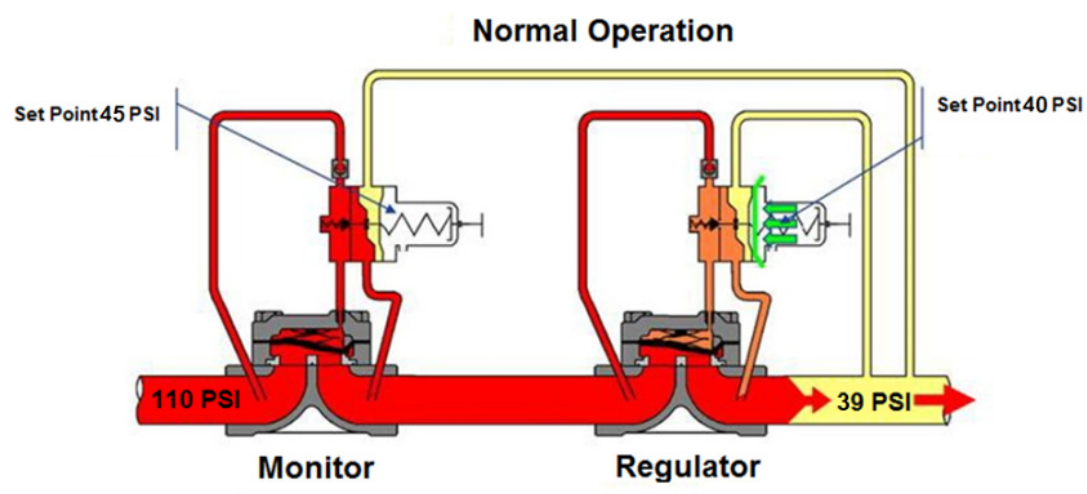

Failure of the Regulator and the Monitor is in control

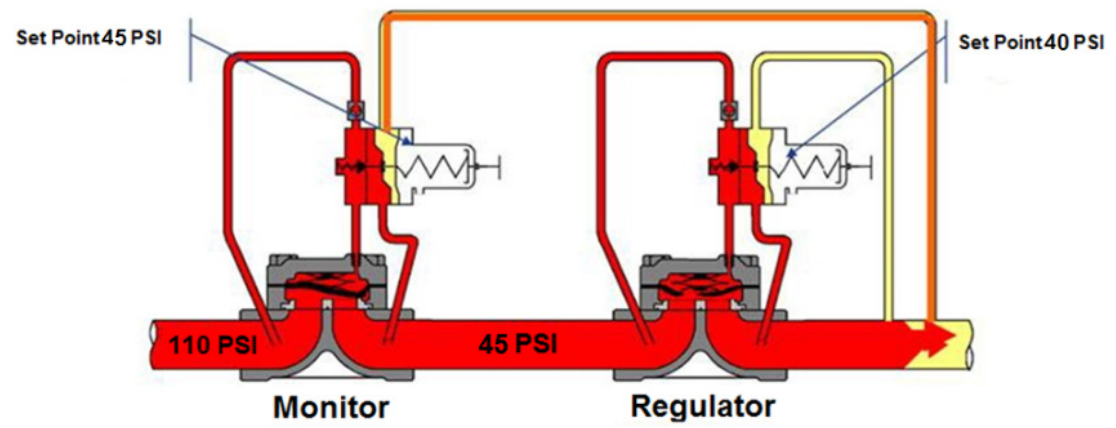

Figure 3. Mooney Regulators in Pressure Reducing Service with a Standby Monitor 


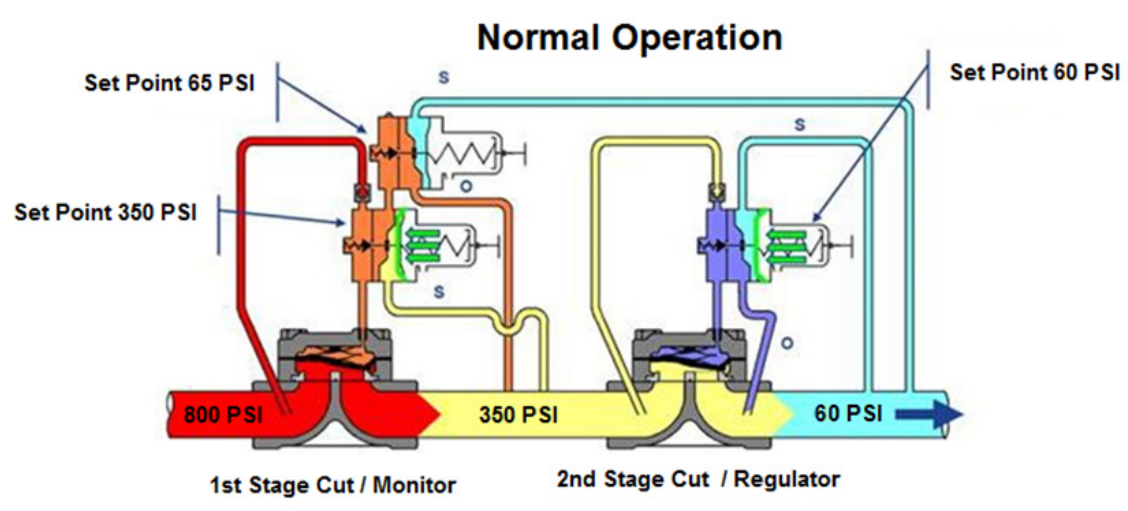

Failure of the Regulator and the monitor in control

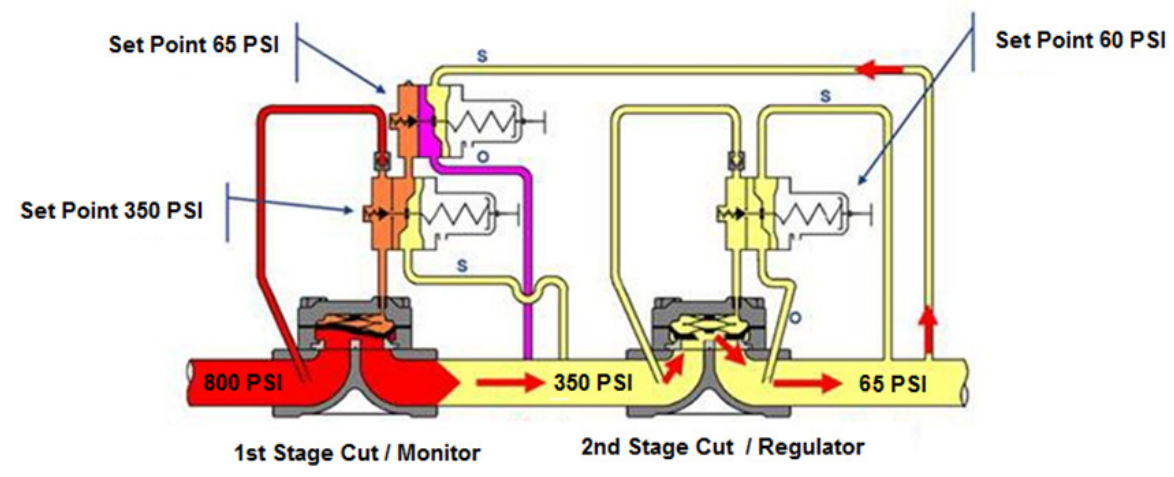

Figure 4. Mooney Regulators in Pressure Reducing Service with a Working Monitor

\subsection{Pressure Regulators with Working Pressure Monitors}

Figure 4 depicts the regulators installed with a working pressure monitor where, a second pressure monitor pilot is used to sense the downstream pressure. Under normal working conditions, the upstream regulator is actively reducing pressure to the inlet of the downstream regulator reducing pressure from $600 \mathrm{psig}$ to $350 \mathrm{psig}$. The downstream regulator is reducing pressure to the established downstream pressure value for the application ( 350 psig to $60 \mathrm{psig}$ ). The monitor pilot is normally set at a pressure value above the second stage regulator value, but below the system Maximum allowable operating pressure. The pressure sensing location is downstream of the second stage regulator. This diagram shows the active control of the pressure monitor when the 2nd stage regulator has failed.

The pressure monitoring criteria for stations with working monitors is the same as those with standby monitors; an upstream transmitter, an intermediate transmitter, and a downstream pressure transmitter are to be installed. The proper function is determined by assessing flow stability and pressure stability for each stage of pressure reduction. Observations of intermediate pressure are key indications of facility integrity.

\subsection{The Standby Monitor Option}

In the set up provided in Figure 4, both regulators are sensing the same downstream pressure. A relatively large pressure drop would occur during demand periods across the regulator while the pressure monitor is wide open. The Pressure drop across the pressure monitor is minimal, and inlet pressure to the regulator would be expected to be nearly the same as the upstream pressure. If the pressure regulator begins to fail, downstream pressure will increase. When the downstream pressure reaches the pressure monitor set point value, the pressure monitor will actively control, this when the gas controller should notice that pressure is now stabilizing at a value greater than it was before the regulator failure. Since gas control will have recorded SCADA data of the pressure monitor set point value, the SCADA system alarm values may be set to advise the gas controller that the pressure monitor is now in control. An active pressure monitor should cause the gas controller to dispatch a service technician to address the regulator issue.

sulfur deposits other contaminants could affect the pressure monitor and might prevent the monitor from opening fully; therefore, monitoring intermediate pressure may provide insights into monitor health and allow a more proactive monitoring of system health. 
Instability of the pressure regulator may be caused by debris in the regulator or maybe the monitor set point being too close to the pressure regulator set point. For either condition, trending downstream pressure and intermediate pressure will be a good indication of the instability problem. Sending a service technician to evaluate the situation is needed in both scenarios to make sure field conditions are evaluated properly.

\subsection{The Working Monitor Option}

In the working monitor configuration, a regulator failure will cause downstream pressure to rise to match the pressure monitor set point values where the pressure monitor takes active control. Intermediate pressure will drop significantly when the pressure reduction is occurring across the pressure monitor. In the case of a pressure monitor failure upstream of the regulator, intermediate pressure will rise such that the entire station differential pressure is taken across the regulator. The rise in intermediate pressure to a value near the upstream pressure is a SCADA alarm function. Intermediate pressure monitoring in these scenarios is important because of the complexity of the first stage regulation and pressure monitor dual functionality.

Our method includes the addition of flow measurement with small profile orifice type flow meter that determines regulator and monitor performance changes. Flow capacity for the station may be compared against current flow rates to see if the regulators are performing properly. For dual or multi-run regulator station as shown in figure 2, flow measurement is critically important to evaluate regulator performance events.

\subsection{Filter Maintenance}

Differential pressure monitoring across the inlet filter element for each regulator run is achieved by installing a pressure switch set to close a contact at a value of 3 psi to 5 psi differential pressure. Given the occurrence of this high differential pressure event, an alarm is set and latched requiring operator reset in the field. This is necessary because the differential pressure will not remain if the filter element fails or if a liquid slug has just passed through the filter element. Either scenario, particulate plugging of the filter or liquid passage, requires an operator to be present on site to remedy the problem and replace the filter element.
The operator would then determine the health of the rest of the facility as well.

\subsection{Water in Underground Regulator Stations}

In the case where the station is below ground in a vault, Water level switches or indicators are installed to advise when levels rise to an unacceptable height. Our proposed heights are making station-piping level as first alarm and at regulators pilots as second and final alarm. Any service of the equipment would probably entail water removal, and a properly equipped maintenance crew would arrive equipped with a pump and tank for water disposal. . In the case of installation of pressure transmitters are sometimes mounted inside the regulator vault, installing level switches is required for proactive intervention before the water level reaches the transmitters, even though they are manufactured to be submerged for limited period of time.

\subsection{Non-Failure Events}

The ideal goal is to install a wide scale number of SCADA with standardized pressure and flow monitoring to allow the development of predictive analysis capabilities. By trending events and discrete pressure values, software and hydraulic models can be created to enable an informed pro-active response to an event prior to any failure event. Example events could be performance instability, unintended regulator interactions or flow limitations due to piping restrictions.

\section{Deductive Analysis by Gas Control}

The goal is to become more proactive in assessing the integrity of regulator installations with the application of the new SCADA installations, achieving operational safety through intelligence capability. Considering facility health, integrity conditions and the observations and actions that the gas control room may take to resolve failure concerns [6][7]. SCADA data to be collected in real time mode thus insuring situation awareness and over time adding situation intelligence with operators seeing and addressing and analyzing repeatability in scenarios, with that predictive analysis [8] [9] will grow enhancing operation intelligence and lowering operational risk (figure 5). 


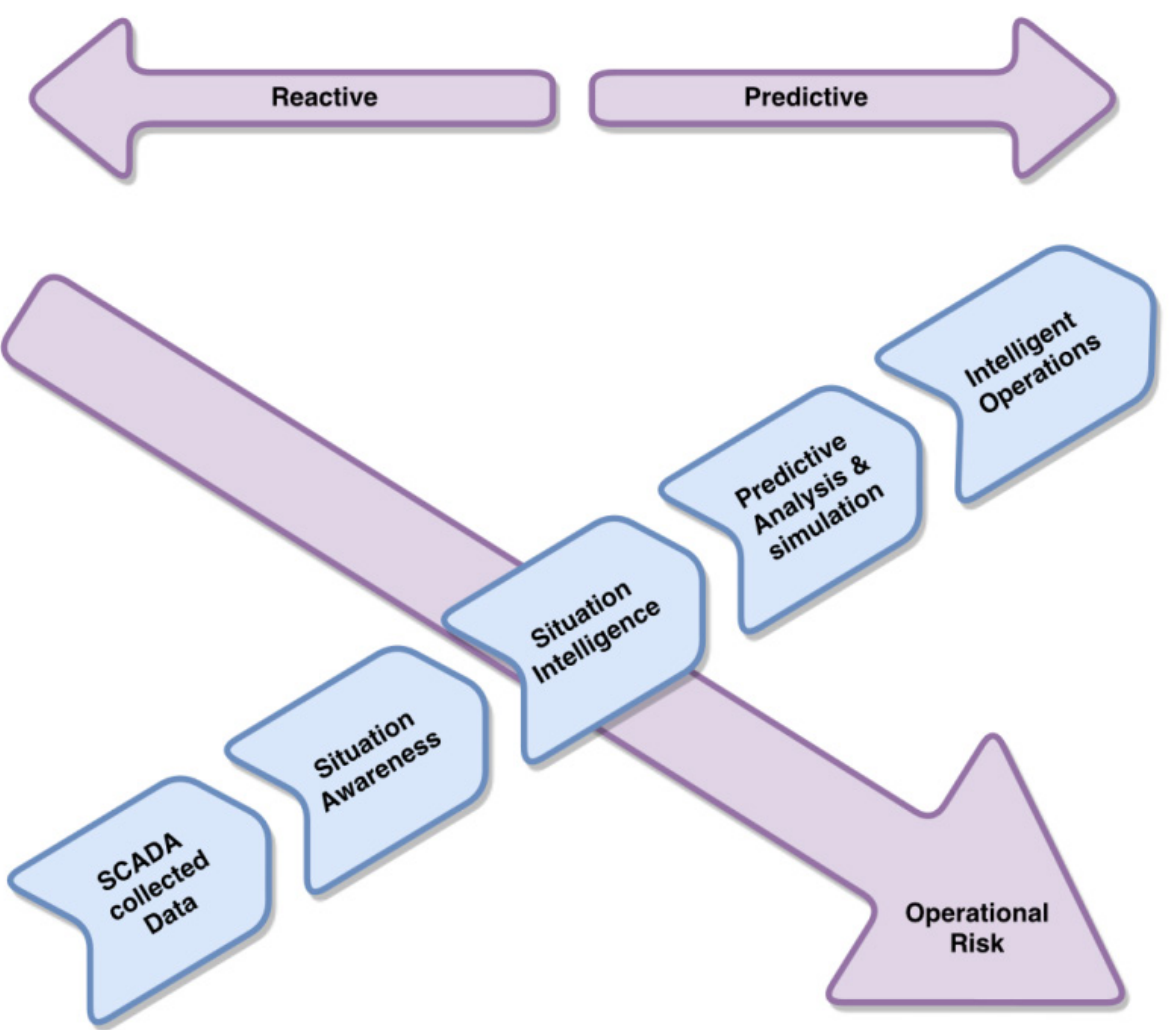

Figure 5. Operational Risk reduction model based on intelligent predictive analysis and operations

\section{Risk Aversion Capabilities}

Figure 6 provides a comprehensive look at the risk aversion provided by each SCADA, functionality we have introduced in this study.

The gas distribution SCADA strategy in the three possible different configurations of regulator stations, Single \& dual run with standby monitor stations, single $\&$ dual run with working monitor and single run with relief valve when and if needed.

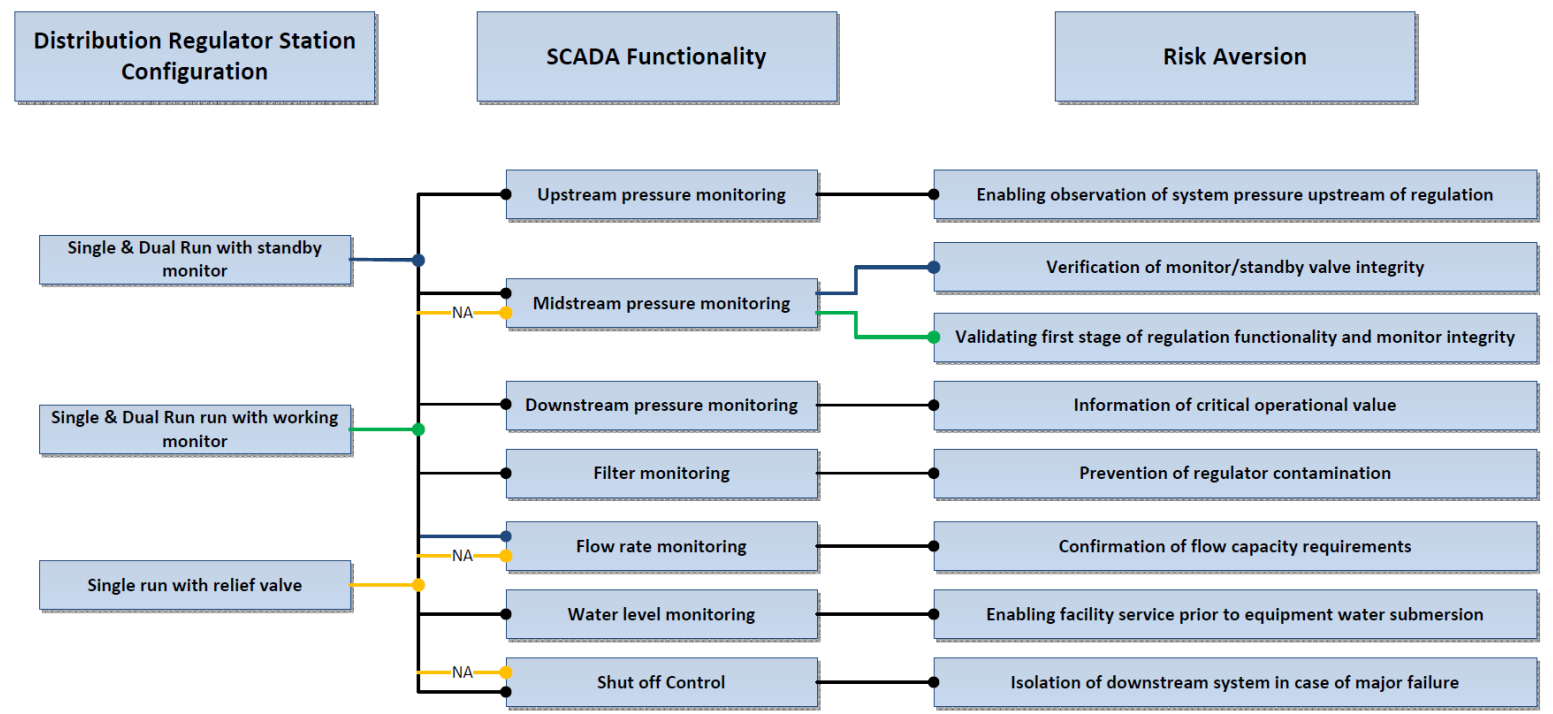

Figure 6. Risk aversion based on SCADA functionality in different configurations of regulator stations 


\section{Conclusions}

The proposed gas distribution SCADA philosophy was evaluated from the perspective of a functional understanding of different pressure reducing regulator and pressure monitor valve installations and modes. It shows different failure modes and the benefits of our SCADA plan by introducing midstream monitoring between the monitor and regular, in addition to Filter pressure differential and water level monitoring in below ground stations.

As shown in our study, enhancing typical industry standard upstream and downstream monitoring with midstream pressure monitoring, filter differential and water level alarms provides more real time and historical data to the gas control room allowing enhanced safety reactivity time and increase predictive capabilities. Collected data over time will allow the creation of robust hydraulic models built around with large sets of real data thus enhancing the capabilities of gas control room in hydraulic systems behavior analysis.

\section{REFERENCES}

[1] N. Kamioka SCADA system of Tokyo Gas for wide-area city gas distribution SICE Annual Conference (SICE), 2012

[2] Yingjie Zhang, Qunjie Xu and Mojie Sun, Application of SCADA System in City Gas Network, Advanced Materials Research (Volumes 616-618). 2012

[3] Annarita Giani, Gabor Karsai, Tanya Roosta, Aakash Shah, Bruno Sinopoli, Jon Wiley, A Testbed for Secure and Robust SCADA Systems. 2008

[4] D. Bailey and E. Wright, Practical SCADA for Industry, Elsevier, 2003

[5] Mooney Flowgrid Regulator - installation, Operation and Maintenance Manual

[6] K. Stouffer, J. Falco and K. Kent, Guide to Supervisory Control and Data Acquisition (SCADA) and Industrial Control Systems Security -Initial Public Draft, National Institute of Standards and Technology, Gaithersburg, Maryland, 2006.

[7] Alan X. L. Zhou, David Yu and Victor Cabrejo, Overpressure Protections in Liquid Pipeline Hydraulic Design - ASME Proceedings | Operations, Monitoring and Maintenance

[8] Predictive analytics in safety and operational risk, Chemicals and Petroleum industries Thought Leadership, 2012

[9] Roberta Bigliani, Reducing Risk in Oil and Gas Operations, IDC Energy Insights \#IDCWP10V 2013 